\title{
Edelfosine lipid nanosystems overcome drug resistance in leukemic cell lines
}

Beatriz Lasa-Saracíbar ${ }^{1}$, Ander Estella-Hermoso de Mendoza ${ }^{1}$, Faustino Mollinedo ${ }^{2}$, María D. Odero $^{3}$, María J. Blanco-Príeto ${ }^{1 *}$

${ }^{I}$ Dept. of Pharmaceutics and Pharmaceutical Technology, School of Pharmacy, University of Navarra, E-31080 Pamplona, Spain

${ }^{2}$ Instituto de Biología Molecular y Celular del Cáncer, Centro de Investigación del Cáncer, CSIC-Universidad de Salamanca, Campus Miguel de Unamuno, E-37007 Salamanca, Spain

${ }^{3}$ Division of Oncology, Center for Applied Medical Research (CIMA), University of Navarra, E31080 Pamplona, Spain

*Corresponding author: Dr. María J. Blanco-Prieto, Department of Pharmaceutics and Pharmaceutical Technology, School of Pharmacy, University of Navarra, C/Irunlarrea 1, E31080 Pamplona, Spain, Office phone: + 34948425600 ext. 6519, Fax: + 34948425 649, email: mjblanco@unav.es 


\begin{abstract}
Although current therapies have improved leukemia survival rates, adverse drug effects and relapse are frequent. Encapsulation of edelfosine (ET) in lipid nanoparticles (LN) improves its oral bioavailability and decreases its toxicity. Here we evaluated the efficacy of ET-LN in myeloid leukemia cell lines. Drug-loaded LN were as effective as free ET in sensitive leukemia cell lines. Moreover, the encapsulated drug overcame the resistance of the K562 cell line to the drug. LN containing ET might be used as a promising drug delivery system in leukemia due to their capacity to overcome the in vivo pitfalls of the free drug and their efficacy in vitro in leukemia cell lines.
\end{abstract}

\title{
Keywords
}

Alkyl-lysophospholipids, edelfosine, lipid nanoparticles, leukemia 


\section{Introduction}

Cancer is one of the leading causes of death worldwide, and the World Health Organization (WHO) estimates that it will cause 13.1 million deaths in 2030 [1]. Among all cancer types, leukemia represents $3 \%$ of total cancer cases [2]. Acute myeloid leukemia (AML) is a heterogeneous clonal disease that disrupts normal hematopoiesis. Leukemic cells are characterized by a block in differentiation and apoptosis, together with an enhanced proliferation. Despite progressive advances in our understanding of the molecular biology of $A M L$, patient outcomes are still very poor. Complete remission occurs in up to half of these patients; however, relapse is generally expected and prognosis is dismal [3]. Therefore, it is necessary to develop more effective treatment strategies to improve the survival of these patients [4]. Among these new therapies, treatment with ether lipids has emerged as a potential alternative to the current ones. Edelfosine (ET) (1-O-octadecyl-2-O-methyl-snglycero-3-phosphocholine) is the prototype of a promising class of anticancer drugs known as synthetic alkyl-lysophospholipids (ALPs), which selectively target tumor cells sparing healthy ones [5]. ET is active against several tumor cell lines [5-7]. The exact mechanism of action of ET still remains to be fully elucidated, but it has been proved that it accumulates in membrane lipid rafts by recruiting and promoting clustering of Fas/CD95 receptors [6,8-10]. Moreover, other apoptotic mechanisms involving mitochondria and endoplasmic reticulum have also been hypothesized [11]. Previous studies have demonstrated that ET induces a rapid apoptotic response in leukemia cells [6]; nevertheless, Tidwell et al. showed that the cell line K562, established from a patient with chronic myeloid leukemia in blast crisis (CML-BC), presents resistance to the action of the drug [12]. Another work by Tsutsumi et al. demonstrated that ET was internalized at a slower rate in ET resistant cells (K562) than in ET sensitive cells (HL-60) [13].

Regardless of promising in vitro results, ET presents some drawbacks when it is administered in vivo, such as gastrointestinal and hemolytic toxicity and low oral bioavailability $[6,14]$. These in vivo disadvantages led to the vehiculization of the drug using nanotechnology [15]. This technology has been widely used in an attempt to improve the therapeutic index of drugs by improving their bioavailability, lowering toxic effects and achieving targeted localization [16]. Among the nanoparticulate systems, lipid nanoparticles (LN) are colloidal carriers that can be produced by an organic solvent free method. Furthermore, these systems have been able to avoid the in vivo drawbacks of ET, diminishing its side effects and increasing its oral bioavailability [17]. Taking this into consideration, the aim of this research was to evaluate the in vitro intracellular uptake and efficacy of drug-loaded LN after administration to sensitive and resistant cell lines.

2. Material and methods

\subsection{Chemicals}

ET was purchased from APOINTECH (Salamanca, Spain). Precirol ${ }^{\circledR}$ ATO 5 was a gift from Gattefossé (France). Tween ${ }^{\circledR} 80$ was purchased from Roig Pharma (Barcelona, Spain). Chloroform was from Panreac (Madrid, Spain), formic acid 99\% for mass spectroscopy was 
obtained from Fluka (Barcelona, Spain), and methanol was purchased from Merck (Barcelona, Spain). All solvents employed for the chromatographic analysis were of analytical grade; all other chemicals were of reagent grade and used without further purification. Amicon Ultra-15 10,000 MWCO centrifugal filter devices were purchased from Millipore (Cork, Ireland). Bovine serum albumin (BSA), trehalose, propidium iodide (PI), RNase, paraformaldehyde and nile red (NR) were acquired from Sigma (Barcelona, Spain). RPMI 1640 and DMEM cell culture media, heat-inactivated fetal bovine serum (FBS) and penicillin/streptomycin antibiotics were purchased from Life Technologies, Invitrogen (Barcelona, Spain). DAPI was obtained from Invitrogen (Madrid, Spain). Fluorescence mounting medium was obtained from Dako (Barcelona, Spain).

\subsection{Preparation and characterization of LN loaded with ET}

LN were prepared by the hot homogenization method consisting of high shear homogenization and ultrasonication [18]. ET (15 mg) and Precirol ${ }^{\circledR}(300 \mathrm{mg})$ were melted at approximately $5^{\circ} \mathrm{C}$ above the melting point of the lipid $(60 \circ)$ ). A $2 \%$ Tween $^{\circledR} 80$ aqueous solution (10 mL) previously heated at the same temperature was added and dispersed in the molten lipid with the help of a Microson ${ }^{\mathrm{TM}}$ ultrasonic cell disruptor (NY, USA) for $1 \mathrm{~min}$ at an effective power of $10 \mathrm{~W}$. The preformed emulsion was then homogenized with an Ultraturrax ${ }^{\circledR}$ (IKA-Werke, Germany) for $1 \mathrm{~min}$ at 24,000 rpm and sonicated again with a Microson ${ }^{\mathrm{TM}}$ ultrasonic cell disruptor (NY, USA) for $1 \mathrm{~min}$ at 10W. The emulsion was removed from heat and placed in an ice bath to obtain LN by lipid solidification. In order to remove the excess of surfactant and non-incorporated drug, the $L N$ suspension was centrifuged using Amicon ${ }^{\circledR}$ Ultra-15 10,000 MWCO filters at $4500 \times \mathrm{g}$ for $30 \mathrm{~min}$ and washed twice with distilled water. Afterwards, $10 \%$ $(\mathrm{w} / \mathrm{v})$ trehalose was added as cryoprotectant agent to the $\mathrm{LN}$ suspension, which was then kept at $-80 \circ \mathrm{C}$ and freeze-dried to obtain a nanoparticulate powder.

Particle size and polydispersity index (PDI) were evaluated by photon correlation spectroscopy (PCS) using a Zetasizer Nano (Malvern Instruments, UK). Each formulation was diluted 30-fold in distilled water in order to avoid multiscattering events. The measurements were carried out three times. Surface charge was measured using the same Zetasizer Nano equipment combined with laser Doppler velocimetry. For the ET loading determination, $5 \mathrm{mg}$ of nanoparticles were dissolved in $1 \mathrm{ml}$ of chloroform and mixed with $4 \mathrm{ml}$ of methanol. The mixture was vortexed for $1 \mathrm{~min}$ and then centrifuged at 20,000 $\times \mathrm{g}$ for $10 \mathrm{~min}$. The supernatant was analyzed by a previously validated ultra-high-performance liquid chromatography tandem mass spectrometry (UHPLC-MS/MS) method [19].

\subsection{Morphology of ET LN}

Morphology of ET LN was observed by transmission electron microscopy (Philips CM120 TEM). Briefly, $5 \mu \mathrm{L}$ of the sample were put on carbon film supported by a copper grid previously submitted to glow discharge and blotted with filter paper to obtain a thin liquid film on the grid. The sample-loaded grid was stained with uranyl acetate for 60 seconds and blotted with filter paper afterwards. The images were recorded with a digital camera (Morada, Olympus).

\subsection{Cell culture}


Four AML cell lines (HL-60, HEL, OCI-AML-2 and MOLM-13) and one CML-BC cell line (K562) were used in this study. HL-60, HEL, MOLM-13 and K562 cell lines were grown in RPMI 1640 culture medium supplemented with $10 \% \mathrm{FBS}, 100$ units $/ \mathrm{mL}$ penicillin and $100 \mu \mathrm{g} / \mathrm{mL}$ streptomycin at $37{ }^{\circ} \mathrm{C}$ in humidified $95 \%$ air and $5 \% \mathrm{CO} 2$. OCI-AML2 was grown in DMEM culture medium supplemented with $10 \% \mathrm{FBS}, 100$ units $/ \mathrm{mL}$ penicillin and $100 \mu \mathrm{g} / \mathrm{mL}$ streptomycin at $37{ }^{\circ} \mathrm{C}$ in humidified $95 \%$ air and $5 \% \mathrm{CO} 2$.

\subsection{Cell proliferation and viability assays}

Cell proliferation and viability were assessed by MTS, included in the CellTiter $96^{\circledR}$ Aqueous One Solution Cell Proliferation Assay (Promega, Madrid, Spain). Cells were treated with culture medium containing various concentrations of free ET, empty nanoparticles (blank-LN) or drugloaded $\mathrm{LN}$ and seeded (100 $\mu \mathrm{L}, 4 \bullet 104$ cells per well) in 96-well culture plates. Plates were incubated at $37^{\circ} \mathrm{C}$ in $5 \% \mathrm{CO}$. After different incubation times (0, 24, 48 and 72 hours), MTS reagent solution was added to culture plate ( $20 \mu \mathrm{L}$ in each well), the mixture was incubated for 2 hours and the formazan production was measured by a microplate spectrophotometer (Labsystems, Helsinki, Finland) at $490 \mathrm{~nm}$ with a reference wavelength of $690 \mathrm{~nm}$.

\subsection{Apoptosis and cell cycle assays}

Apoptosis Staining with Annexin-V-Fluos Kit (Roche, Madrid, Spain) was used for these experiments. Cells were treated with culture medium containing various concentrations of free-ET, blank-LN or drug-loaded $\mathrm{LN}$ and seeded in 12-well culture plates $(4 \mathrm{~mL}, 1.6 \bullet 106$ cells per well).

Quantification of apoptotic cells after each treatment was performed by flow cytometry using Annexin-V-FLUOS Staining $\mathrm{Kit}^{\circledR}$ following the manufacturer's instructions. Cells were harvested $(500 \mu \mathrm{L})$, centrifuged at $200 \times \mathrm{g}$ for $5 \mathrm{~min}$ at $20^{\circ} \mathrm{C}$ and washed with PBS. Afterwards, they were suspended in $100 \mu \mathrm{L}$ of Annexin-V-FLUOS labeling solution. This solution was incubated for 15 min at room temperature, $300 \mu \mathrm{L}$ of the incubation buffer were added to each cytometer tube after the incubation time and samples were analyzed on a FacsCalibur flow cytometer (Becton Dickinson, NJ, USA).

For the cell cycle analysis, cells were harvested $(500 \mu \mathrm{L})$, centrifuged at $200 \times \mathrm{g}$ for $5 \mathrm{~min}$ at $20^{\circ} \mathrm{C}$ and washed twice with PBS. Cells were then treated with ice-cold ethanol $70 \%$ in order to fix them and permeabilize their membrane; samples were then washed with PBS and suspended in a mixture of $445 \mu \mathrm{L}$ of PBS, $50 \mu \mathrm{L}$ of PI and $5 \mu \mathrm{L}$ of RNase per sample. Finally, cells were incubated in darkness for 1 hour and analyzed by flow cytometry.

\subsection{LN uptake}

NR-loaded LN were formulated by adding the fluorochrome $(0.34 \% \mathrm{w} / \mathrm{w})$ to the lipid phase (method described in section 2.2). HL-60 and K562 cells were treated with culture medium containing various concentrations of free-ET, blank-LN or NR-LN and seeded in 24-well culture plates $(500 \mu \mathrm{L}, 3 \bullet 105$ cells per well). After different incubation times, cells were collected by centrifugation, washed twice with PBS and analyzed in a flow cytometer. For the microscopy studies, cells were fixed with $p$-formaldehide $4 \%$ (300 $\mu \mathrm{l}, 5 \mathrm{~min})$. They were then stained with DAPI for $5 \mathrm{~min}$. Samples were then placed on 24-well plates with a cover glass on the surface 
of the wells. Cells were adhered to the surface of the cover glass by centrifugation of the plate at $260 \times \mathrm{g}$ for $5 \mathrm{~min}$. Finally, cover glasses were extracted and placed on microscope slides with fluorescence mounting media. Samples were examined on a fluorescence microscope (Zeiss, 120 Libra).

\subsection{Caspase-3/8/9 detection}

Caspase 9 and 3 activities were detected by CaspGlowTM Red Active Caspase-9 Staining Kit and CaspGlowTM Fluorescein Active Caspase-3 Staining Kit, respectively (BD Biosciences, Madrid, Spain). Caspase 8 activity was detected by cleaved Caspase-8 (Asp 391) (18C8) (Cell Signaling, Barcelona, Spain). HL-60 and K562 cells were treated with culture medium containing various concentrations of free-ET, blank-LN or drug-loaded LN and seeded in 24well culture plates $(500 \mu \mathrm{L}, 5 \bullet 105$ cells per well). After different incubation times, cells were incubated with the antibody (caspase-3/9) provided in the kits following the manufacturer's instructions. For the caspase- 8 assay, cells were fixed with $\mathrm{p}$-formaldehyde, permeabilized with methanol (90\%) and washed twice with a solution of BSA $(0.5 \%)$. Antibody was then added and incubated for 1 hour at room temperature. Samples were then washed twice with incubation buffer and analyzed by flow cytometry.

\subsection{Statistical analysis}

Each experiment was repeated three times $(n=3)$ and the average of the three values was used for statistical calculations. Each experimental value was expressed as mean \pm standard deviation (SD). Statistical analyses were performed using GraphPad Prism 5. Groups that are significantly different from control are indicated in the figures as * $p<0.05, * * p<0.01$ and $* * * p<0.001$.

\section{Results and discussion}

Nanotechnology applications in medicine have improved the therapeutic efficacy of many anticancer drugs [16]. The encapsulation of the ether lipid ET into LN has led to a reduction of its in vivo toxicity and to an increase in its oral bioavailability [17]. These promising in vivo results, along with the great efficacy of the free drug shown in vitro in myeloid leukemia [5], opened up the possibility of applying this formulation to this disease. This study aimed to investigate the in vitro antitumor effect of $E T$ in $L N$ versus the free drug in myeloid leukemia cells (HL-60, HEL, OCI-AML-2, MOLM-13 and K562).

\subsection{LN containing ET: physicochemical characterization.}

The average size was close to $100 \mathrm{~nm}$, suitable for oral administration with a PDI index less than 0.3 in all cases, suggesting homogenous distribution of the nanoparticulate suspension (Table 1). Blank-LN presented a size $20 \mathrm{~nm}$ over drug-loaded LN. This difference may be attributed to the tensoactive properties of the drug given by its amphiphilic nature. LN charge was negative and enough to maintain nanoparticle stability [20]. TEM images (Fig. 1) confirmed the size of LN previously measured by PCS. The LN obtained showed good encapsulation efficiency of $75 \%$, presenting a drug loading of $13 \mu \mathrm{g}$ ET per mg of formulation. 
3.2. ET antitumor effect in sensitive cell lines is preserved when using encapsulated drug, while drug activity in the resistant cell line is enhanced when it is included in LN.

Previous in vitro studies have shown that leukemia cells have different sensitivities to the antitumor action of ET [5]. Hence, we aimed to classify the cell lines used in this work into sensitive and resistant cells, in order to reveal the advantage of encapsulating the drug. MTS proliferation assay was used to obtain the IC50 of free and encapsulated drug in the different leukemia cells. Comparison of IC50 values of free ET and drug-containing LN (Table 2) led to the classification of the cell lines in two groups based on their sensitivity to the free drug: sensitive cell lines (HL-60, OCI-AML-2, MOLM-13 and HEL) and resistant (K562). HL-60, MOLM13, HEL and OCI-AML-2 showed similar low IC50 values that were, in all cases, less than $5 \mu \mathrm{M}$ when treated with free drug, OCl-AML-2 being the most sensitive to the action of the free drug with an IC50 of $0.64 \mu \mathrm{M}$. LN were more efficient in OCI-AML-2 and MOLM-13 and less efficient in HL-60 and HEL. Nevertheless, IC50 values of nanoencapsulated drug were less than $9 \mu \mathrm{M}$ in all sensitive cell lines. To further clarify these results, Figure 2 shows dose-response curves of ET and ET-LN in MOLM-13, selected as representative sensitive cell line, and in K-562 resistant cell line. As it can be seen, the dose-response curve for the sensitive cell line is similar showing comparable IC50 values for ET and ET-LN. In contrast to the sensitive cell line, ET-LN were able to produce a pronounced cell growth inhibition in the resistant $\mathrm{K} 562$ cell line compared to free ET. Indeed, the IC50 of ET in this cell line, was $20 \mu \mathrm{M}$ for the drug-loaded LN compared to $57.70 \mu \mathrm{M}$ of the free ET (Figure 2 and Table 2).

Cell proliferation was measured by MTS reagent, which is bioreduced by metabolically active cells. Proliferation studies were carried out in all the cell lines at different drug concentrations over a period of $72 \mathrm{~h}$. The efficacy of drug-loaded LN in inhibiting cell growth was similar to the free drug in sensitive cell lines (HL-60, MOLM-13, OCI-AML-2 and HEL). Both treatments showed fast and potent inhibition of cell proliferation, achieving in some cases a complete inhibition of cell viability after 72 hours of incubation. In these cell lines, free and drug-loaded $\mathrm{LN}$ were effective at the lowest dose $(5 \mu \mathrm{g} / \mathrm{ml} ; 9.5 \mu \mathrm{M})$ used in the study. The initial effect of the free drug is faster in sensitive cell lines probably due to the time needed by the encapsulated drug to be internalized and released into the cell; indeed, the antitumor effect is similar at the end of the treatment with both treatments. In resistant cell line K562, free ET showed a slight inhibition effect, whereas encapsulated drug was able to inhibit proliferation from the first 24 hours of incubation at a $L N$ dose equivalent to $10 \mu \mathrm{g} / \mathrm{ml}(19 \mu \mathrm{M})$ of free drug. This overcoming of resistance might be related to an increased ET concentration inside the cell after LN uptake; it also could be associated with an enhanced apoptotic mechanism due to the different intracellular localization of the drug. These results prove that encapsulating the drug in LN does not affect drug activity in sensitive leukemia cells; moreover, nanoencapsulated ET was able to overcome the resistance to the free drug in the case of resistant cells.

Cytotoxicity of LN containing ET versus the free drug was analyzed by flow cytometry. Viable, early apoptotic, late apoptotic and necrotic cells can be distinguished by flow cytometric analysis using dual staining with annexin V-FITC and PI dyes. Viable cells are negative for both stains; early apoptotic cells bind annexin due to the expression of phosphatidylserine in the outer-leaflet of cell membrane and late apoptotic cells bind both stains because PI only binds DNA when the cell membrane losses its integrity. Our data showed that ET induced cell death 
in a dose- and time-dependent manner. As in proliferation studies, free ET was able to induce apoptosis earlier than the encapsulated drug in sensitive cell lines; Fig. 3 shows the induction of apoptosis by the different treatments in OCIAML-2 cells after 24 and 72 hours of incubation. However, the percentages of apoptotic cells produced by both treatments were equivalent after 72 hours of incubation, being the percentage of viable cells in OCI-AML-2 less than $10 \%$ (Fig. 3). Figure 4 shows the percentage of apoptotic cells in sensitive cells after 72 hours of incubation with both treatments (free and nanoencapsulated drug); these results prove that encapsulating the drug in LN does not affect its apoptotic effect. In K562 cells, free ET did not show any apoptotic effect; in contrast, drug-loaded LN were able to diminish the number of living cells at a dose equivalent to $15 \mu \mathrm{g} / \mathrm{ml}(28.6 \mu \mathrm{M})$ of ET. Figure 5 shows that drug-loaded LN were able to decrease significantly the percentage of viable cells with respect to the untreated cells after 72 hours of incubation with the treatment.

The cell cycle is a tightly regulated process in normal cells and cancer is closely related to cell cycle abnormalities. Cell cycle steps are deregulated due to mutations in proto-oncogenes and tumor suppressor genes [21]. In cancer cells, cell cycle checkpoints are altered and cells undergo uncontrolled growth. Many antitumor drugs act at this level inducing cell arrest and preventing cell division. As a result, cell cycle analysis was performed to examine whether the inhibition of cell proliferation and apoptosis induction involved cell cycle changes. The results obtained are in accordance with previous studies that affirm that ET inhibits cell division leading to the accumulation of cells into G2/M [22]. ET induces a G2/M cell cycle arrest increase percentage of dead cells in sensitive cells (Fig. 6). As it was observed in apoptosis studies, free drug effect was faster but both treatments achieved similar death rates at the end of the treatment in sensitive cells (Fig. 7). The analysis of $\mathrm{K} 562$ cell cycle revealed that, again, drug-loaded LN were effective inducing cell cycle arrest in G2/M phase (Fig. 6). Free-ET also induces this phase arrest; however, cell cycle arrest might not be enough to induce apoptosis and cell cycle analysis also shows that only loaded LN increase the percentage of cells in Sub G1 peak (dead cells) in resistant cells. This led to the conclusion that only the encapsulated drug is able to trigger the whole machinery of apoptotic mechanisms in resistant cells.

3.3. NR-LN are internalized quickly and in a time-dependent manner in sensitive and resistant cell lines.

In contrast to free ET, which interacts with the cell membrane and accumulates in lipid rafts [23], LN require endocytosis to release the drug inside the cell [24]. This process might change the antitumor activity of the drug because ET LN might target the drug to a different intracellular localization. ET cell entry occurs through membrane lipid rafts [23]; however, drug-loaded LN might have a different intracellular localization after nanoparticle entry. Nanoparticle uptake by leukemia cells was studied incorporating a lipophilic fluorochrome into the LN. NR was added to the lipid phase and LN were formulated following the same method as for ET-loaded LN. NR-LN showed similar size, PDI and charge to drug-loaded LN. Drugloaded NR-LN were also formulated confirming that NR does not affect ET encapsulation. However, drug-loaded NR-LN charge was $-14.9 \mathrm{mV}$ and, therefore, blank-LN were used to study nanoparticle internalization on the basis of their similarity to the original formulation (Table 1). To evaluate LN uptake, HL-60 (sensitive) and K562 (resistant) cells were incubated 
with NR-LN for different times. The NR-LN internalization was analyzed by fluorescence microscopy and flow cytometry (Fig. 8 and 9). The data reported here show that LN were internalized into leukemia cancer cells very rapidly and in a time-dependent manner in both cell lines. This internalization might be independent of cell sensitivity to the free drug since it is incorporated in the LN. This could mean that, in contrast to free ET [13], encapsulated drug might easily be incorporated into $\mathrm{K} 562$ cells enhancing its intracellular concentration, which could trigger a major response to the drug. Interestingly, Fig. 8 shows how LN accumulate in the cell membrane after one hour of incubation indicating a strong interaction between the particles and cells. Furthermore, red fluorescence was all over the cytoplasm avoiding the nucleus at later incubation times. Unfortunately, the extended diffusion of the fluorochrome avoided the colocalization inside the cytoplasm with different organelle labels, thus hampering the determination of the exact localization the nanoparticles inside the cell. These results are consistent with previous uptake studies of LN by Teskac and Kristl [25] that show the rapid internalization and diffuse localization of LN containing 6-coumarin dye in keratinocytes. Nanoparticle uptake was quantified by flow cytometry to obtain a quantitative analysis of NRLN internalization. The results showed accordance with fluorescence microscopy studies. Nanoparticles were internalized in a time-dependent manner achieving $70 \%$ of cells containing fluorescent nanoparticles after 3 hours of incubation (Fig. 8). This internalization pattern was also observed in resistant cells (Fig. 9) confirming the microscopy results.

\subsection{Free and encapsulated ET induce Caspases-3, 8 and 9 only in sensitive cells.}

The exact mechanism of action of ET still remains remains to be fully elucidated; however, it has been seen that there are many different mechanisms implicated. Intrinsic and extrinsic apoptotic signaling pathways are considered the major programmed mechanisms of cell death. Both processes are mediated by cysteine proteases named caspases. The intrinsic apoptosis pathway is mediated by mitochondria, and caspase- 9 is considered the predominant initiator caspase. The extrinsic pathway is triggered from the cell membrane and it is mediated by death receptors, such as Fas/CD95 receptor. In this case, signaling cascade is mainly initiated by caspase- 8 cleavage. Both apoptotic mechanisms converge in the induction of caspases-3/7 [26].

Recent studies suggest that ET mainly accumulates in membrane lipid rafts and triggers the extrinsic apoptotic pathway throughout Fas/CD95 death receptor $[9,10,23,27]$. However, this mechanism might be diminished in the case of encapsulated drug, as it could be directed to different cell targets once inside the cell, promoting other drug actions and therefore overcoming resistance to the drug. In fact, it has also been described that ET also triggers the intrinsic apoptotic signaling pathway $[23,28]$. Besides, there are more mechanisms implicated such as endoplasmic reticulum stress and the production of reactive oxygen species $[29,30]$. To gain further insight into mechanisms for apoptosis induction, HL-60 and K562 cells were incubated with free and drug-loaded LN and samples were analyzed after different incubation times by flow cytometry to detect activated caspases. Free ET and LN containing the drug induced the cleavage of caspase- 8 and 9 and, subsequently, also induced caspase- 3 in HL-60 cells (Fig. 10). This caspase induction in sensitive leukemia cells occurs with free and 
encapsulated drug but it is faster when cells are treated with free ET. Caspase induction was time-dependent becoming more pronounced after 72 hours of incubation. However, no caspase induction was observed in $\mathrm{K} 562$ cells regardless of the treatment. Bearing in mind that drug-loaded LN induce apoptosis mediated death as was demonstrated by flow cytometry analysis; these data suggest the existence of caspase independent apoptosis pathways triggered by encapsulated ET. In fact, other mechanisms such as oxygen reactive species production have already been described as ET antitumor mechanism [29,30]. Drug-loaded LN might be able to overcome the resistance of $\mathrm{K} 562$ cells to the entrance of the free drug. This increased influx of drug into the cell increases its apoptotic mechanism overcoming drug resistance. Among the apoptotic mechanisms that might be involved in the loaded LN effect, we have demonstrated that encapsulated drug triggers a G2/M peak arrest in cell cycle. Although free-ET also induces a cell cycle arrest, this effect is not able to induce apoptosis by itself. Therefore, loaded LN might be able to induce multiple apoptotic mechanisms that finally overcome free drug resistance.

\section{Conclusions}

The present research allows us to conclude that $L N$ are potential vehicles of ET in myeloid leukemia. LN are internalized in sensitive and resistant leukemia cells possibly overcoming the difficulties of the free ET in being internalized in K562 cells. LN preserve the potent apoptotic effect that free ET has in sensitive leukemia cells by means of caspase activation. Nanoparticles could be triggering other pathways of programmed cell death that appear to be independent from caspase activation in edelfosine-resistance cells.

\section{Acknowledgements}

This work has been carried out in the framework of the COST Action TD1004. Financial support from Caja Navarra Foundation, University of Navarra (FUN), the Government of Navarra, Department of Health (ref: 63/09, 'Ortiz de Landázuri' fellowship), the Spanish Ministry of Science and Innovation (SAF2010-15547, SAF2011-30518) and RD06/0020/1037 from 'Red Temática de Investigación Cooperativa en Cáncer', Instituto de Salud Carlos III, cofunded by the 'Fondo Europeo de Desarrollo Regional' of the European Union is acknowledged. B. LasaSaracibar is supported by the research grant from "Asociación de Amigos de la Universidad de Navarra".

References

[1] WHO: Cancer, 2012. Available at: http://www.who.int/mediacentre/factsheets/fs297/en/ [last accessed 09 October 2012] 
[2] Iarc: Globocan 2008 fast stats. Available at:

http://globocan.iarc.fr/factsheets/populations/factsheet.asp?uno=900 [last accessed 09

October 2012]

[3] J.M. Rowe, Optimal induction and post-remission therapy for AML in first remission. Hematology, Am Soc Hematol Educ Program. 1 (2009) 396-405.

[4] T. Haferlach, Molecular genetic pathways as therapeutic targets in acute myeloid leukemia, Hematology Am Soc Hematol Educ Program 1 (2008) 400-411.

[5] F. Mollinedo, J.L. Fernández-Luna, C. Gajate, B. Martín-Martín, A. Benito, R. MartinezDalmau, M. Modolell, Selective induction of apoptosis in cancer cells by the ether lipid ET-18$\mathrm{OCH} 3$ (edelfosine): Molecular structure requirements, cellular uptake, and protection by bcl-2 and bcl-X(L), Cancer Res. 57 (1997) 1320-1328.

[6] F. Mollinedo, J. de la Iglesia-Vicente, C. Gajate, A. Estella-Hermoso de Mendoza, J.A. VillaPulgarín, M. de Frias, G. Roué , J. Gil , D. Colomer, M.A. Campanero, M.J. Blanco-Prieto, In vitro and in vivo selective antitumor activity of edelfosine against mantle cell lymphoma and chronic lymphocytic leukemia involving lipid rafts, Clin. Cancer Res. 16 (2010) 2046-2054.

[7] F. Mollinedo, J. de la Iglesia-Vicente, C. Gajate, A. Estella-Hermoso de Mendoza, J.A. VillaPulgarin, M.A. Campanero, M.J. Blanco-Prieto, Lipid raft-targeted therapy in multiple myeloma, Oncogene 26 (2010) 3748-3757

[8] F. Mollinedo, C. Gajate, Fas/CD95 death receptor and lipid rafts: New targets for apoptosisdirected cancer therapy, Drug Resist Updat. 9 (2006) 51-73.

[9] C. Gajate, F. Mollinedo, The antitumor ether lipid ET-18-OCH3 induces apoptosis through translocation and capping of Fas/CD95 into membrane rafts in human leukemic cells, Blood. 98 (2001) 3860-3863.

[10] C. Gajate, E. del Canto-Jañez, A.U. Acuña, F. Amat-Guerri, E. Geijo, A.M. Santos-Beneit, R.J. Veldman, F. Mollinedo, Intracellular triggering of Fas aggregation and recruitment of apoptotic molecules into Fas-enriched rafts in selective tumor cell apoptosis, J Exp Med 200, (2004) 353365.

[11] C. Gajate, M. Matos-da-Silva, E.L. Dakir, R.I. Fonteriz, J. Alvarez, F. Mollinedo, Antitumor alkyl-lysophospholipid analog edelfosine induces apoptosis in pancreatic cancer by targeting endoplasmic reticulum, Oncogene 31 (2012) 2627-2639

[12] T. Tidwell, G. Guzman, W.R. Vogler, The effects of alkyl-lysophospholipids on leukemic cell lines. I. Differential action on two human leukemic cell lines, HL60 and K562, Blood. 57 (1981) 794-797.

[13] T. Tsutsumi, A. Tokumura, S. Kitazawa, Undifferentiated HL-60 cells internalize an antitumor alkyl ether phospholipid more rapidly than resistant K562 cells, Biochim. Biophys. Acta. 1390 (1998) 73-84. 
[14] C. Gajate, F. Mollinedo, Biological activities, mechanisms of action and biomedical prospect of the antitumor ether phospholipid ET-18-OCH3 (edelfosine), a proapoptotic agent in tumor cells, Curr Drug Metab 3, (2002) 491-525.

[15] A. Estella-Hermoso de Mendoza, M. Rayo, F. Mollinedo, M.J. Blanco-Prieto, Lipid nanoparticles for alkyl lysophospholipid edelfosine encapsulation: Development and in vitro characterization, Eur. J. Pharm. Biopharm. 68 (2008) 207-213.

[16] A. Estella-Hermoso de Mendoza, B. Lasa-Saracíbar, M.A, Campanero, M.J. Blanco-Prieto, Lipid Nanoparticles in Biomedicine, Encyclopaedia of Nanoscience and Nanotechnology, American Scientific Publishers, USA, 2010, pp 455-478.

[17] A. Estella-Hermoso de Mendoza, M.A. Campanero, H. Lana, J.A. Villa-Pulgarin, J. de la Iglesia-Vicente, F. Mollinedo, M.J. Blanco-Prieto, Complete inhibition of extranodal dissemination of lymphoma by edelfosine-loaded lipid nanoparticles, Nanomedicine UK. 7 (2012) 679-690.

[18] A. Estella-Hermoso de Mendoza, V. Preat, F. Mollinedo, M.J. Blanco-Prieto, In vitro and in vivo efficacy of edelfosine-loaded lipid nanoparticles against glioma, J. Control. Release. 3 (2011) 421-426.

[19] A. Estella-Hermoso de Mendoza, M. A. Campanero, F. Mollinedo, M. J. Blanco-Prieto, Comparative study of a HPLC-MS assay versus an UHPLC-MS/MS for anti-tumoral alkyl lysophospholipid edelfosine determination in both biological samples and in lipid nanoparticulate systems, J. Chromatogr. B. Analyt Technol. Biomed. Life. Sci. 877 (2009) 40354041.

[20] S. Das, A. Chaudhury, Recent advances in lipid nanoparticle formulations with solid matrix for oral drug delivery, AAPS PharmSciTech. 12 (2011) 62-76.

[21] F. McLaughlin, P. Finn, N.B. La Thangue, The cell cycle, chromatin and cancer: Mechanismbased therapeutics come of age, Drug Discov. Today. 8 (2003) 793-802.

[22] K. P. Boggs, C. O. Rock, S. Jackowski, Lysophosphatidylcholine attenuates the cytotoxic effects of the antineoplastic phospholipid 1-O-octadecyl-2-O-methyl-rac glycero-3phosphocholine, J Biol Chem 270 (1995) 270-11612.

[23] C. Gajate, F. Mollinedo, Edelfosine and perifosine induce selective apoptosis in multiple myeloma by recruitment of death receptors and downstream signaling molecules into lipid rafts, Blood. 109 (2007) 711-719.

[24] G. Sahay, D.Y. Alakhova, A.V. Kabanov, Endocytosis of nanomedicines, J. Control. Release. 145 (2010) 182-195.

[25] K. Teskac, J. Kristl, The evidence for solid lipid nanoparticles mediated cell uptake of resveratrol, Int. J. Pharm. 390 (2010) 61-69.

[26] B. Favaloro , N. Allocati1, V. Graziano, C. Di llio, V. De Laurenzi, Role of Apoptosis in disease, Aging 4 (2012) 330-349 
[27] C. Gajate, F. Mollinedo, Lipid rafts and Fas/CD95 signaling in cancer chemotherapy, Recent. Pat. Anticancer Drug Discov. 6 (2011) 274-283.

[28] C. Gajate, A. M. Santos-Beneit, A. Macho, M. Lazaro, A. Hernandez-De Rojas, M. Modolell, E. Muñoz, F. Mollinedo, Involvement of mitochondria and caspase-3 in ET-18-OCH(3)-induced apoptosis of human leukemic cells, Int. J. Cancer. 86 (2000) 208-218.

[29] H. Zhang, C. Gajate, L.P. Yu, Y.X. Fang, F. Mollinedo, Mitochondrial-derived ROS in edelfosine-induced apoptosis in yeasts and tumor cells, Acta Pharmacol Sin. 28 (2007) 888894.

[30] V.A. Selivanov, P. Vizán, F. Mollinedo, T.W. Fan, P.W. Lee, M. Cascante, Edelfosine-induced metabolic changes in cancer cells that precede the overproduction of reactive oxygen species and apoptosis, BMC Syst Biol. 4 (2010) 135.

Table 1

Physicochemical characteristics of the developed LN (mean \pm SD)

Formulation

Size $(\mathrm{nm})$

$P D I$ $\zeta$-potential

$E E(\%)$

Drug loading

$(m V)$

( $\mu g \mathrm{drug} / \mathrm{mg}$ 


\begin{tabular}{cccccc}
\hline & \multicolumn{1}{c}{ formulation) } \\
\hline Blank-LN & $123.80 \pm 9.02$ & $0.260 \pm 0.015$ & $-28.1 \pm 2.4$ & -- & - \\
Drug-loaded LN & $104.83 \pm 3.40$ & $0.248 \pm 0.006$ & $-26.5 \pm 5.6$ & $75.75 \pm 10.26$ & $13.02 \pm 0.99$ \\
& & & & -- & -- \\
NR-LN & $117.53 \pm 1.58$ & $0.246 \pm 0.010$ & $-21.5 \pm 0.4$ & & $11.87 \pm 1.52$ \\
\hline
\end{tabular}


Table 2.

$\mathrm{IC}_{50}$ values of leukemia cell lines alter $72 \mathrm{~h}$ of incubation with free-ET and Drug-loaded LN.

\begin{tabular}{cccc} 
Cell type & Cell name & Free-ET $(\mu M)$ & Loaded LN $(\mu M)$ \\
\hline Human acute myeloid leukemia & HL-60 & 3.48 & 8.64 \\
Human acute myeloid leukemia & OCI-AML-2 & 0.64 & 0.22 \\
Human acute myeloid leukemia & MOLM-13 & 3.64 & 3.41 \\
Human erythroleukemia & HEL & 4.42 & 7.96 \\
Human chronic myeloid leukemia & K562 & 57.70 & 20 \\
\hline
\end{tabular}


(a)

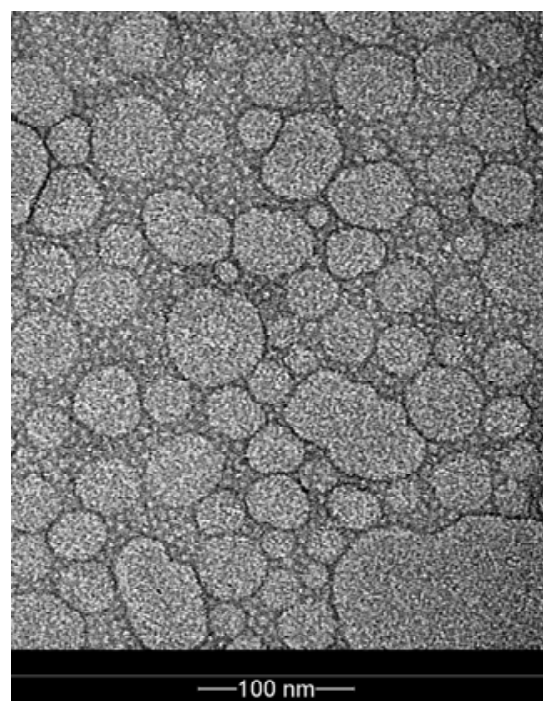

(b)

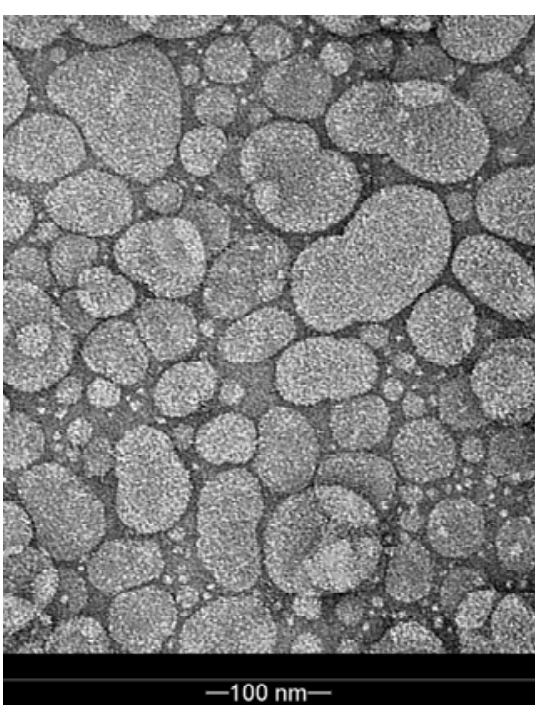

Figure 1. TEM images of blank-LN (a) and Drug-loaded LN (b). 

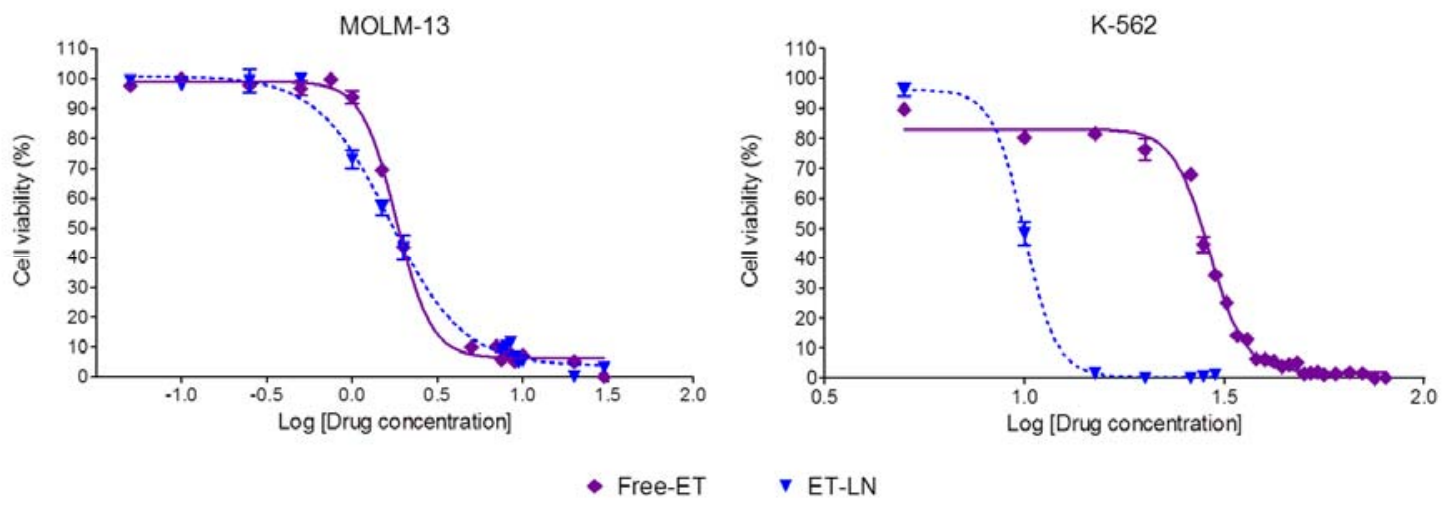

Figure 2. Dose- reponse curve in sensitive (MOLM-13) and resistant (K-562) cells at 72 hours of incubation with different doses of free ET or drug-loaded LN. 

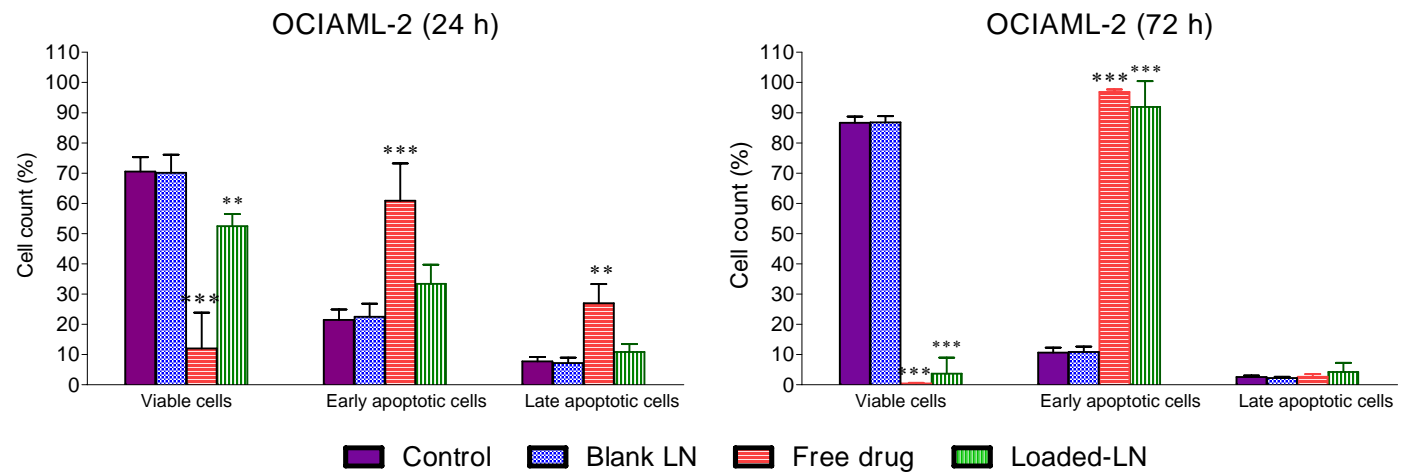

Figure 3. Apoptosis induced in OCI-AML-2 (sensitive cells) by the different treatments (9.5 $\mu \mathrm{M}$ of ET) after $24 \mathrm{~h}$ and $72 \mathrm{~h}$ of incubation. ${ }^{* *} \mathrm{p}<0.01$; ${ }^{* *} \mathrm{p}<0.001 \mathrm{vs}$. control by two-way ANOVA (Bonferroni post-test). 

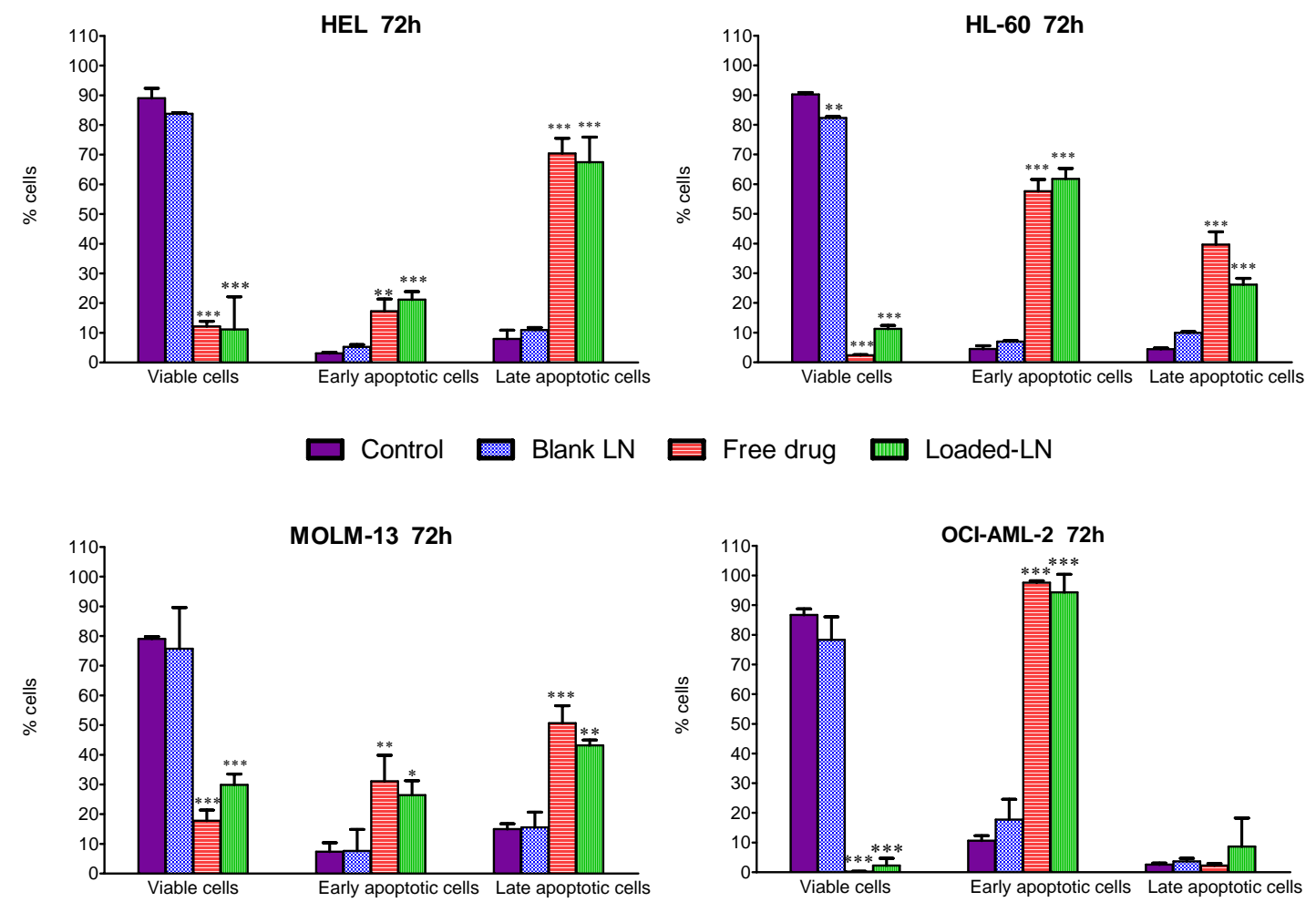

Figure 4. Apoptosis induced in sensitive cell lines by the different treatments (19 $\mu \mathrm{M}$ of ET) after 72 h of incubation. ${ }^{*} \mathbf{p}<0.05 ;{ }^{* *} \mathbf{p}<0.01 ;{ }^{* * *} \mathbf{p}<0.001$ vs. control by two-way ANOVA (Bonferroni posttest). 

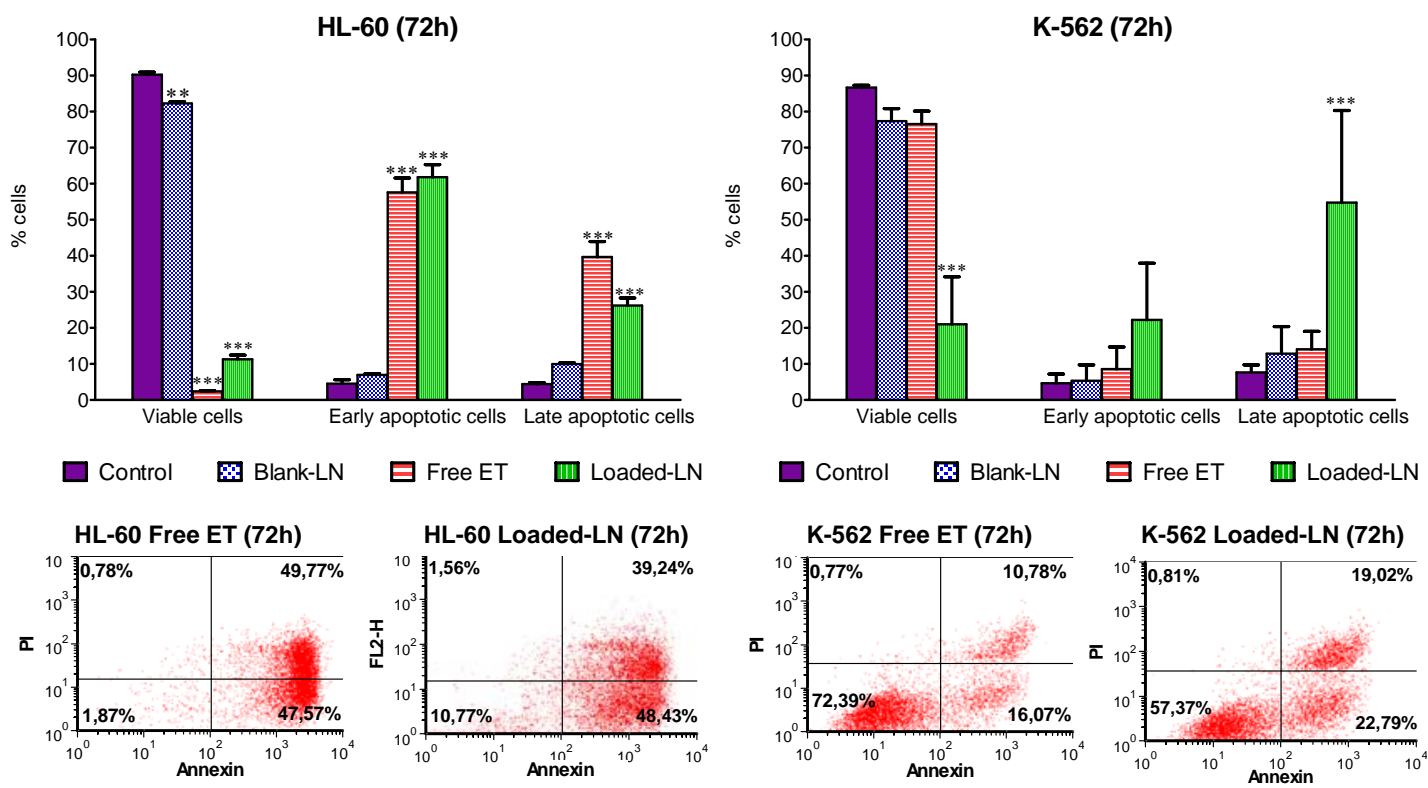

Figure 5. Flow cytometry graphs of apoptosis induced in HL-60 and K562 cells after 72 hours of incubation with medium, blank-LN, free ET and drug-loaded $L N$ at a dose equivalent to $19 \mu M$ (HL-60) and $28.6 \mu \mathrm{M}$ (K562) of free ET. ${ }^{*} \mathbf{p}<0.05$; ${ }^{* *} \mathbf{p}<0.01$; ${ }^{* * *} \mathbf{p}<0.001$ vs. control by two-way ANOVA (Bonferroni post-test). 

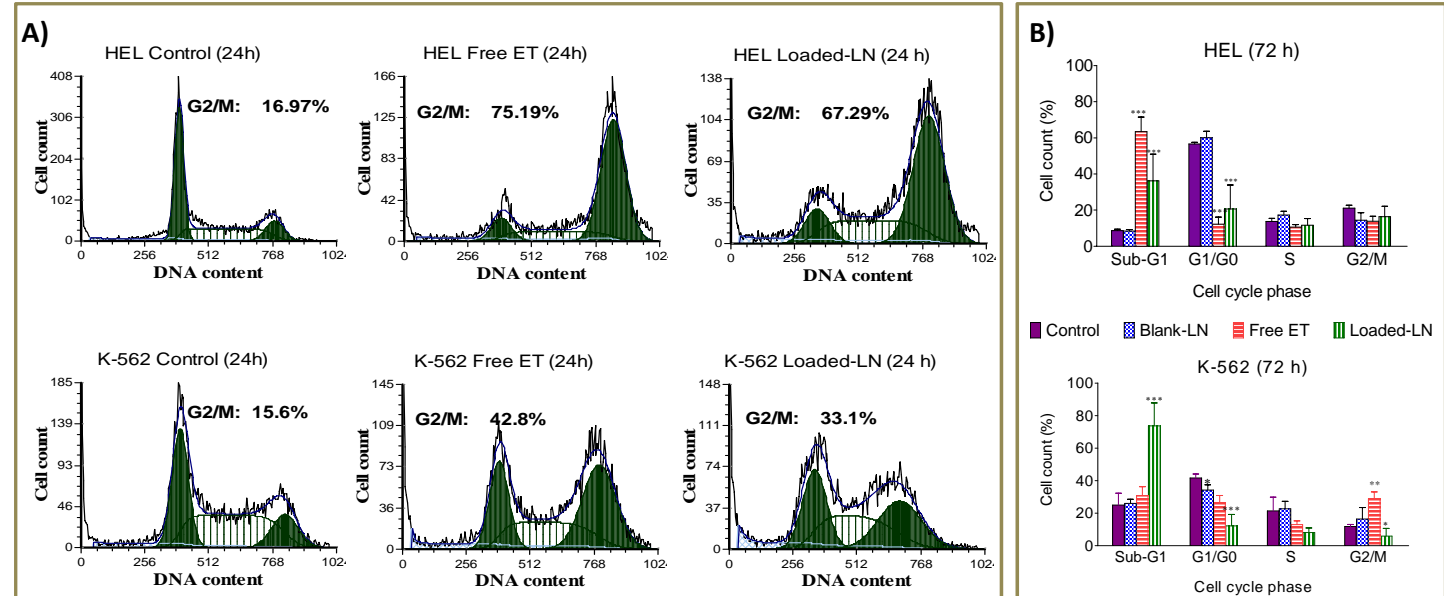

Figure 6. (A) Free and nanoencapsulated drug effect in cell cycle distribution in sensitive (HEL) and resistant (K562) cells after 24 (A) and 72 (B) hours of incubation. Cells were treated with medium, blank-LN, free ET and drug-loaded LN at a dose equivalent to $9.5 \mu \mathrm{M}$ of ET (HEL) and $28.6 \mu \mathrm{M}$ of ET (K562). * $\mathbf{p}<0.05 ;{ }^{* *} \mathbf{p}<0.01$; ${ }^{* * *} \mathbf{p}<0.001$ vs. control by two-way ANOVA (Bonferroni post-test). 

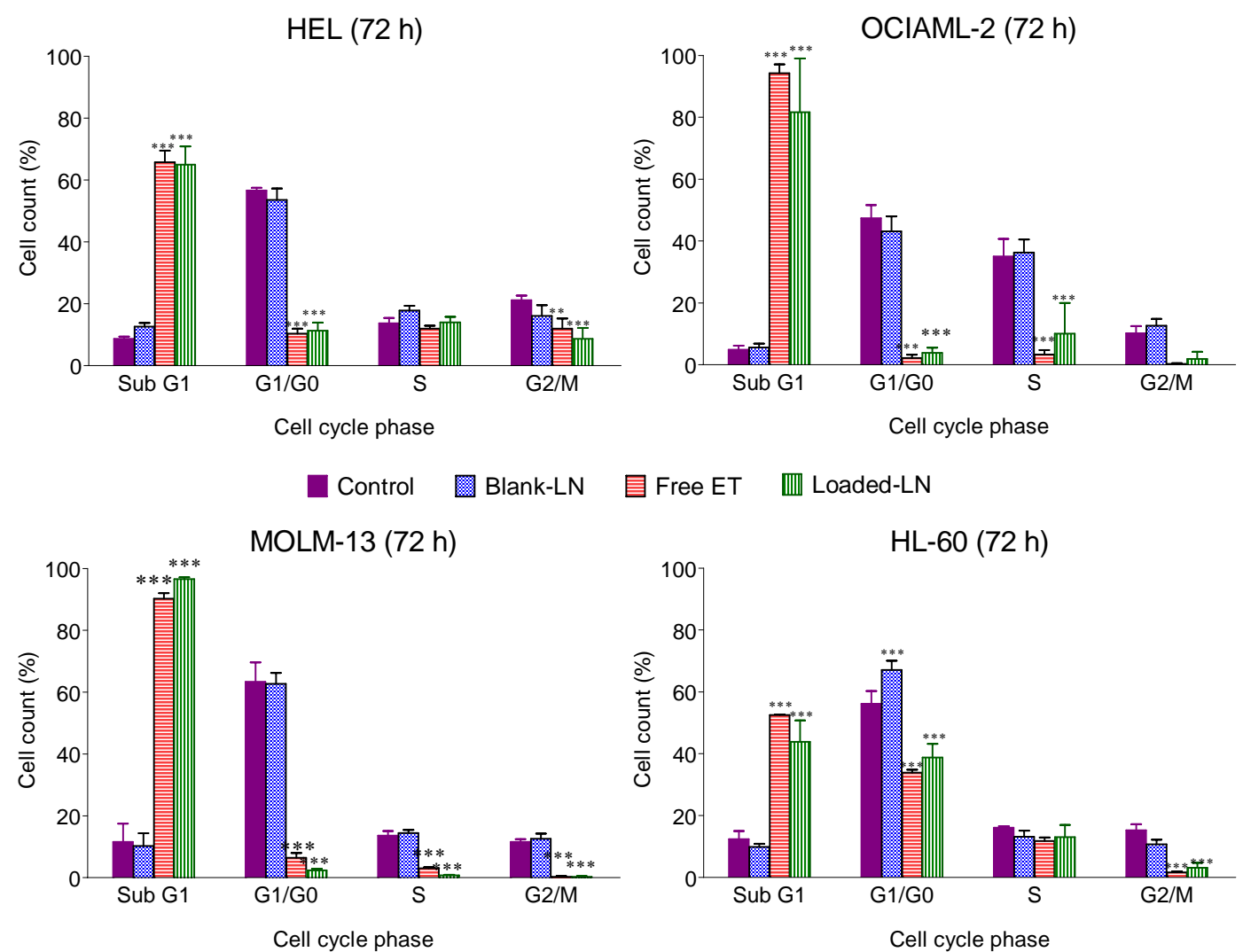

Figure 7. Analysis of cell cycle distribution in sensitive cell lines by the different treatments (at a dose corresponding to $19 \mu \mathrm{M}$ of ET) after $72 \mathrm{~h}$ of incubation. ${ }^{*} \mathbf{p}<0.05 ;{ }^{*} \mathbf{p}<0.01 ; * * * \mathbf{p}<0.001 \mathrm{vs}$. control by two-way ANOVA (Bonferroni post-test). 


\section{HL-60 (sensitive cell line)}

A)

$20 x$

Nile Red LN

MERGED

(Nile Red LN+ DAPI)

Oh

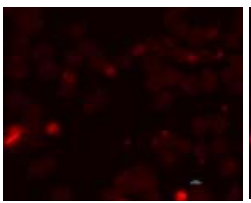

$1 \mathrm{~h}$

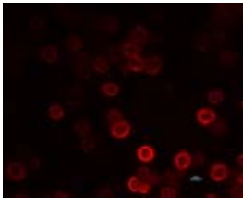

$6 h$

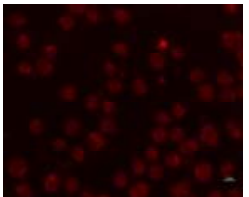

$24 h$

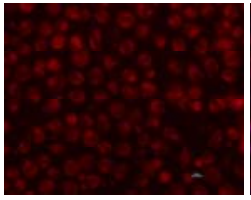

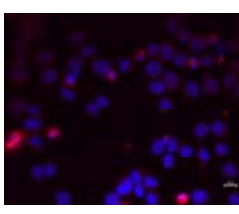
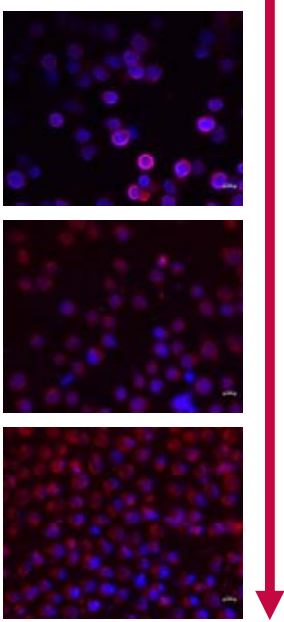

B)

Nile Red LN internalization after 3 hours of incubation

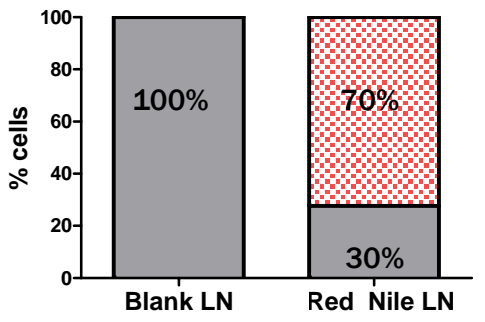

$\square \%$ Negative Nile Red cells

$\square$ \% Positive Nile Red cells

C)

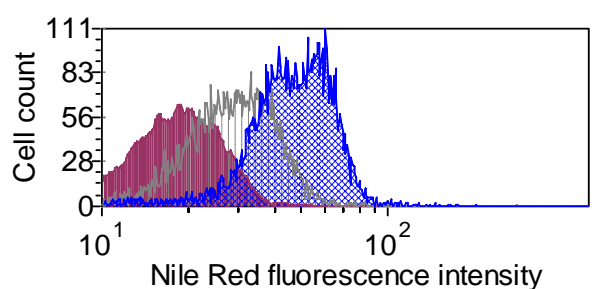

NR-LN Oh NR-LN 1h NR-LN 3h

Figure 8. (A) Fluorescence images of HL-60 cells cultured with NR-LN for different incubation times. (B) Flow cytometry graph corresponding to NR-LN uptake by HL-60 cells after 3 hours of incubation with NR-LN. (C) Flow cytometry analysis of NR-LN uptake within the time (3 hours) after incubation of HL-60 cells with NR-LN. 


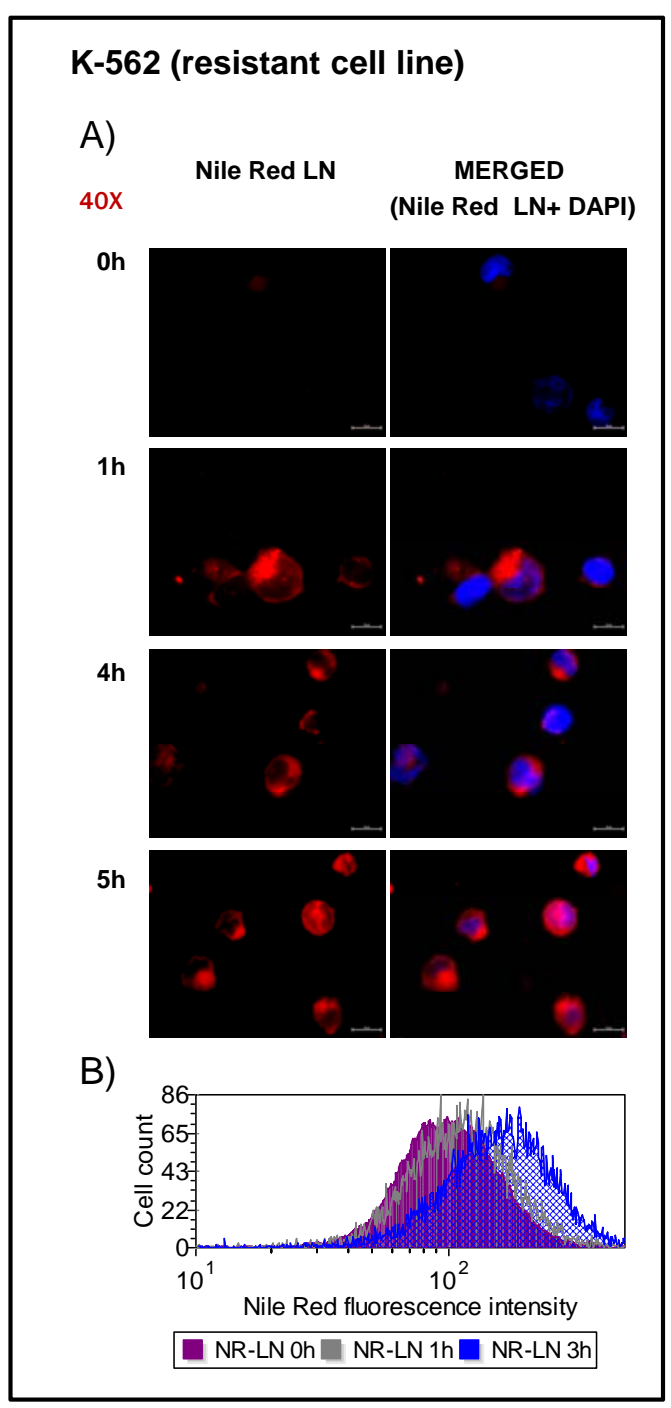

Figure 9. (A) Fluorescence images of K562 cells cultured with NR-LN for different incubation times. (B) Flow cytometry graph corresponding to NR-LN uptake by K562 cells within the time (3 hours). 

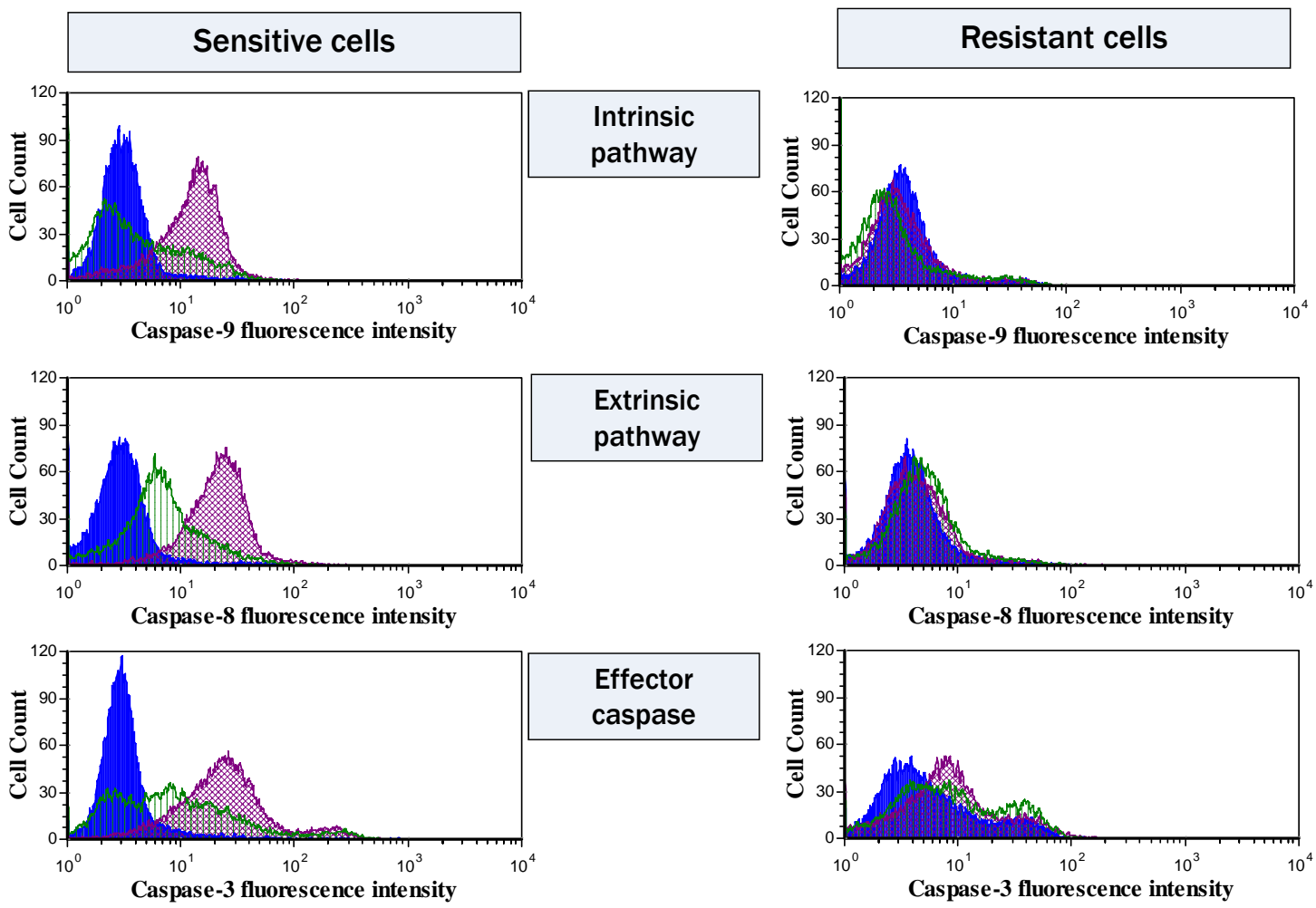

$$
\text { Control } \bigotimes \text { Free ET W Loaded LN }
$$

Figure 10. Caspase-9, 8 and 3 activation in HL-60 (sensitive) and K562 (resistant) cells by flow cytometry. Cells were untreated (control), treated with free ET (Free-ET) or drug-loaded LN (Loaded-LN) with a dose equivalent to $19 \mu \mathrm{M}$ free ET and analyzed after 72 hours of incubation. 